\title{
High-Expression of Cytoplasmic Poly (A) Binding Protein I (PABPCI) as a Prognostic Biomarker for Early-Stage Esophageal Squamous Cell Carcinoma
}

\author{
Jiangtao $\mathrm{Pu}^{\prime}$ \\ Tao Zhang (D) \\ Dengguo Zhang' \\ Kaiming $\mathrm{He}^{\prime}$ \\ Yonghong Chen ${ }^{2}$ \\ Xingwang Sun ${ }^{3}$ \\ Wenbo Long $\mathbb{1}^{3}$ \\ 'Thoracic Surgery Department of the \\ First Affiliated Hospital, Southwest \\ Medical University, Sichuan, People's \\ Republic of China; ${ }^{2}$ Laboratory of \\ Affiliated Hospital of traditional Chinese \\ medicine of Southwest Medical \\ University, Sichuan, People's Republic of \\ China; ${ }^{3}$ Pathology Department of the \\ First Affiliated Hospital, Southwest \\ Medical University, Sichuan, People's \\ Republic of China
}

Background and Objective: Poly (A) binding protein cytoplasmic 1 (PABPC1) plays a crucial role in the regulation of RNA polyadenylation, translation initiation, and mRNA stability and may be involved in tumorigenesis. Herein, we set out to identify the prognostic value of PABPC1 expression in esophageal squamous cell carcinoma (ESCC).

Methods: Using quantitative real-time PCR (qRT-PCR) and immunohistochemical analysis, the present study investigated mRNA and protein expressions of PABPC1 in 231 ESCCs and their paired adjacent normal epithelial tissues.

Results: We observed a reduction in the average mRNA expression of $P A B P C 1$ in ESCC tissue specimen, but the mRNA expression of $P A B P C 1$ was significantly higher $(\mathrm{P}<0.001)$ in ESCC tissues with high PABPC1 expression and lower $(\mathrm{P}=0.033)$ in tissues with low PABPC1 expression. In immunohistochemical analysis, positive expression of the PABPC1 protein was identified in 179 ESCC tissue specimens (179/231, 77.5\%), while the percentage of ESCC tissue specimens with high expression of PABPC1 was found to be $41.1 \%(95 / 231)$. PABPC1 expression was found to be significantly correlated with lymph node metastasis (LNM) $(\mathrm{P}=0.011)$, pathological stage $(\mathrm{P}=0.021)$, tumor recurrence $(\mathrm{P}<0.001)$, and the outcome $(\mathrm{P}<0.001)$ of patients with ESCC. High expression of PABPC1 was associated with poor overall survival $(\mathrm{OS})$ of ESCC patients $(\mathrm{P}<0.001)$ among all pathological stages, particularly in the early stages (pStage-I and -II), and identified to be an independent prognostic factor for OS of patients with ESCC in multivariate analysis $(\mathrm{HR}=2.622$; 95\% CI, 1.68-4.129). Comparatively, the expression of Ki-67, p53, and nm23 was not associated with OS.

Conclusion: In this study, we discovered that PABPC1 is a prognostic biomarker and a therapeutic target for ESCC, particularly early-stage ESCC.

Keywords: esophageal squamous cell carcinoma, cytoplasmic poly (A) binding protein 1, immunohistochemical staining, overall survival, prognostic factor, hazard ratio

\section{Introduction}

Esophageal cancer is an aggressive malignant tumor that develops from the inner lining (mucosa) of the esophagus and is associated with a reduced overall outcome. It represents the eighth most frequently diagnosed malignancy worldwide and the sixth leading cause of cancer-associated mortality. ${ }^{1}$ Esophageal squamous cell carcinoma (ESCC) represents the predominant histological sub-types of esophageal cancer, and accounts for over $70 \%$ of total esophageal cancer cases worldwide. ${ }^{2}$ Diagnosis of ESCC at an early stage is difficult. ESCC exhibits local invasion and
Correspondence: Wenbo Long

Email wenbolong@swmu.edu.cn 
lymph node metastasis (LNM) at the advanced stage and even at initial diagnosis, thus, contributing to the poor prognosis and low survival rate. ${ }^{3,4}$ Advances in new diagnostic techniques, surgical techniques, and chemotherapy have not substantially improved its prognosis over the last two decades. ${ }^{5}$ Therefore, identifying the mechanisms underlying ESCC tumorigenesis and developing new effective biomarkers to improve ESCC diagnosis and prognosis remains highly desirable.

The mRNA persistence and degradation plays an essential role in the control of gene expression. Alterations in the control of mRNA stability may have a profound impact on cell growth, cell-cycle control, and tumorigenesis. ${ }^{6}$ Cytoplasmic poly (A) binding protein 1 (PABPC1), the major cytoplasmic isoform of poly(A) binding proteins, is believed to play an essential role in mRNA stabilization and translation enhancement. PABPC1 interacts with mRNA, pre-initiation factors, and other regulators to form complexes that promote translation while safeguarding the mRNA from exonucleases. $^{7-9}$ Moreover, PABPC1 functions in the mechanisms of miRNA mediated silence and nonsensemediated decay (NMD) mRNA surveillance. ${ }^{10}$ Thus, the post-transcriptional regulation of gene expression provided by PABPC1 plays an extremely important role in regulating most cellular processes. Evidence suggests that the dysregulation in the expression of PABPC1 is associated with tumorigenesis in a variety of malignant tumors. For example, overexpression of PABPC1 has been reported in gastric carcinoma tissues and hepatocellular carcinoma, particularly in high-grade tumors, ${ }^{11,12}$ while the reduced mRNA level of PABPCl has been positively associated with the survival time in ESCC. ${ }^{13}$ However, the prognostic value of PABPC1 in ESCC remains elusive.

Herein, we aimed to uncover the relationship between PABPC1 expression and ESCC, and to discover the efficacy of PABPC1 as a biomarker to predict survival of ESCC patients after undergoing an esophagectomy. Through the use of quantitative real-time PCR (qRTPCR) and immunohistochemical (IHC) analysis, the present study investigated mRNA and protein expressions of PABPC1 in ESCCs and their paired adjacent normal epithelial tissues. The results indicated that PABPC1 protein expression was markedly up-regulated in ESCC tissues and localized to the cytoplasm. Moreover, overexpression of PABPC1 was significantly associated with advanced stage, LNM, and poor prognosis.

\section{Methods}

\section{Patients and Specimens}

In total, 231 primary ESCC tissues and paired adjacent normal epithelium tissues were obtained from patients who underwent a pathological diagnosis and surgical resection for ESCC at the First Affiliated Hospital of Southwest Medical University (Luzhou, China) between January 2012 and March 2015. For this study, only histopathologically confirmed ESCC cases were included. Besides, patients with other malignancies or those received chemo- or radiotherapy before surgery were excluded from the study. Of these, there were 200 men and 31 women with a median age of 61.0 years (range, 43-79 years) (Supplementary Table 1). All patients' data in this manuscript were coded and utilized anonymously.

The specimens were fixed in $10 \%$ formaldehyde and embedded in paraffin. The paraffin-samples were then stored at the Department of Pathology, Southwest Medical University at room temperature. The ESCC and normal tissues were cut into $4 \mu \mathrm{m}$ sections and stained with standard hematoxylin-eosin (H\&E) stain for histopathological evaluation. Pathological tumor node metastasis (TNM) classification and corresponding staging were defined following the criteria proposed by the seventh edition American Joint Committee on Cancer (AJCC). This study was conducted in accordance with the Declaration of Helsinki and approved by the Institutional Research Ethics Committee of the First Affiliated Hospital of Southwest Medical University, China (No. KY2019126) and written informed consent was obtained from all patients. Researchers did not have access to any identifying information for any of the patients or samples in this study. The clinical endpoints were overall survival (OS) defined as the time from the first surgical resection of ESCC until death or the end of follow-up (12/31/2020). All patients were followed up regularly after surgery; however, 79 (34.2\%) patients were lost to follow-up as these patients were from remote areas, and the contact was very inconvenient.

\section{RNA Extraction, Reverse Transcription, and Real-Time PCR (RT-PCR)}

Total RNA was extracted from the paraffin-embedded cancer and normal tissue through the use of total RNA extraction kit for paraffin-embedded tissue sections (Tin Gen biochemical technology (Beijing) Co., Ltd, Item: DP439), as per the manufacturer's protocol. The RNA 
quality was determined ultilizing AmoyDx SMA4000 Bioanalyzer. Only RNA with a $28 \mathrm{~S}: 18 \mathrm{~S}$ ratio $>1.8$ and an OD range of 1.85-2.10 were used for subsequent analysis. The cDNA was reverse transcribed using the Promega M-MLV reverse transcriptase, as per the manufacturer's protocol. Real-time fluorescent qPCR was conducted with the Promega GoTaq qPCR and RT-qPCR systems in a Roche quantitative PCR thermocycler. The reaction comprised 1 cycle at $95^{\circ} \mathrm{C}$ for $3 \mathrm{~min}$, followed by 45 cycles of $95^{\circ} \mathrm{C}$ for $15 \mathrm{sec}$ and $60^{\circ} \mathrm{C}$ for $40 \mathrm{sec}$. The $2-\Delta \Delta \mathrm{CT}$ method was used to determine relative gene expression, which was normalized to the amount of GAPDH mRNA. All experiments were performed at least in triplicate for each gene. The primers that were used to detect the mRNA expression of PABPC1 and GAPDH (the reference gene) are as follows. The primers for $P A B P C 1$ are f-GCCAGTACGCATCATGTGGTCTC and r-CATACAGTGCTTTATTATCAATGG. The GAPDH primers are f-GCTTGTCATCAATGGAAATCCC and r-GGAGGGATCTCGCTCCTGG.

\section{Immunohistochemical Analysis}

For IHC analysis, paraffin-embedded ESCC tissues and paired normal tissues were serially sectioned $(4-\mu \mathrm{m})$, heated at $60^{\circ} \mathrm{C}$ for $1 \mathrm{~h}$, and subjected to IHC. The sections were deparaffinized in xylene and then rehydrated in graded ethanol series. Subsequently, slides were submerged into an endogenous peroxidase blocking solution containing 3\% hydrogen peroxide and methanol for 10 min, and were then washed with phosphate-buffered saline (PBS) for 2 min and blocked with 5\% goat serum for $30 \mathrm{~min}$ at RT. Antigen retrieval was achieved by transferring the sections in citrate buffer ( $\mathrm{pH}$ 6.0) (for $\mathrm{nm} 23$ and PABPC1) or EDTA buffer (for Ki-67 and p53). Next, sections were incubated with primary antibodies, including PABPC1 antibody (1:1000; ab21060; Abcam); mouse monoclonal Ki-67 antibody (1:200; MAB-0672), mouse monoclonal p53 antibody (1:200; MAB-0674), and mouse monoclonal nm23 antibody (1:200; MAB-0139) from Maixin Biotechnology Co. (Fuzhou, China), and incubated overnight at $4^{\circ} \mathrm{C}$. Subsequently, the sections were stained for protein detection in 3,3-diaminobenzidine (DAB) system and counterstained with Mayer's hematoxylin. As the negative control, the primary antibody was omitted, and sections were incubated with corresponding secondary antibodies and detection systems. Stained sections were examined under a microscope.
The expression status of PABPC1 immunostaining was reviewed independently by two pathologists who were blinded to clinical data. Only heavy intensity staining within the tumor region was recorded as a positive stain. Next, the percentage of positive tumor cells relative to the total number of tumor cells was calculated. High expression of PABPC1 was defined as $\geq 50 \%$ positive staining on the slide and low expression of PABPC 1 was $<50 \%$. For $\mathrm{Ki}-67$ analysis, proliferation index of $\mathrm{Ki}-67$ was calculated as number of positive cells divided by total number of examined cells among five selected $200 \times$ fields. ${ }^{14}$ For p53 and $\mathrm{nm} 23$, the expression of the biomarker was evaluated according to percentage of positive tumor cells. Tumor cell percentage of $<5 \%$ and $<10 \%$ for p 53 and $\mathrm{nm} 23$, respectively, were defined as negative expression, while the percentage of $\geq 5 \%$ and $\geq 10 \%$, respectively, as positive. ${ }^{15,16}$

\section{Statistical Analysis}

Data were expressed as the mean \pm standard deviation. Continuous variables were presented as percentages and analyzed using the $t$-test, and categorical variables were analyzed using the $\chi^{2}$ test. Survival curves for OS analysis were estimated using the Kaplan-Meier method, and the significant differences between the survival curves were compared using a Log rank test. Independent predictors related to the OS of patients with ESCC were first analyzed using univariate analysis. Significantly associated variables identified by univariate analysis were entered into a Cox proportional hazards regression model using the backward elimination method for multivariate analysis. Hazard ratio (HR) and 95\% confidence interval (CI) were estimated. All statistical tests were two-tailed, and a P-value $<0.05$ was considered statistically significant $(* p<0.05, * * p<0.01)$. All statistical analyses were performed using SPSS version 19.0 (IBM Armonk, NY, USA).

\section{Results}

\section{Patients' Clinicopathological Characteristics}

This study included a total of 231 patients with ESCC comprising 200 men (86.6\%) and 31 women (13.4\%) with a medium age of 61 years (ranged 43-79) (Table 1). Chronic alcoholism and smoking were more frequently found among men with ESCC than among women patients $(\mathrm{P}<0.001)$. The occurrence of ESCC was detected at higher levels in the lower esophagus but at lower levels 
Table I Clinicopathologic Parameters of the 23I ESCC Patients

\begin{tabular}{|c|c|c|c|c|}
\hline Variable & Total & Man & Woman & $P$ \\
\hline Age (y), median (range) & $\begin{array}{l}61.0(43-79) \\
n=231\end{array}$ & $\begin{array}{l}60.7(43-79) \\
n=200\end{array}$ & $\begin{array}{l}62.7(47-78) \\
n=31\end{array}$ & 0.169 \\
\hline Smoking, $>5 /$ day (\%) & $79 / 134(59.0)$ & $78 / 117(66.7)$ & I/I7 (5.9) & $<0.001 * *$ \\
\hline Alcohol, >25 g/day (\%) & $78 / 133(58.6)$ & $76 / 116(65.5)$ & $2 / 17(11.8)$ & $<0.001 * *$ \\
\hline Tumor site, U/M/L (\%) & $\begin{array}{l}18 / 99 / 99 \\
(8.3 / 45.8 / 45.8) \\
n=216\end{array}$ & $\begin{array}{l}14 / 82 / 93 \\
(7.4 / 43.4 / 49.2) \\
n=189\end{array}$ & $\begin{array}{l}4 / 17 / 6 \\
(14.8 / 63.0 / 22.2) \\
n=27\end{array}$ & $0.026 *$ \\
\hline Tumor size, median (range) & $\begin{array}{l}3.9(1.1-15.0) \\
n=231\end{array}$ & $\begin{array}{l}4.0(1.1-8.5) \\
n=200\end{array}$ & $\begin{array}{l}3.9(1.5-15.0) \\
n=31\end{array}$ & 0.765 \\
\hline DTCD, well/moderate/low (\%) & $\begin{array}{l}18 / 110 / 103 \\
(7.8 / 47.6 / 44.6) \\
n=231\end{array}$ & $\begin{array}{l}16 / 94 / 90 \\
(8 / 47 / 45) \\
n=200\end{array}$ & $\begin{array}{l}2 / 16 / 13 \\
(6.5 / 51.6 / 41.9) \\
n=31\end{array}$ & 0.879 \\
\hline LN & & & & \\
\hline No. of LNs diagnosed, median (range) & $\begin{array}{l}16.1(1-54) \\
n=231\end{array}$ & $\begin{array}{l}16.6(1-54) \\
n=200\end{array}$ & $\begin{array}{l}12.9(4-28) \\
n=31\end{array}$ & 0.620 \\
\hline No. of LNs metastasized, median (range) & $\begin{array}{l}I .48(0-2 I) \\
n=23 I\end{array}$ & $\begin{array}{l}1.54(0-21) \\
n=200\end{array}$ & $\begin{array}{l}1.06(0-6) \\
n=31\end{array}$ & 0.974 \\
\hline Pathological type, ME/UT/MU/CT (\%) & $\begin{array}{l}44 / 153 / 19 / 8 \\
(19.6 / 68.3 / 21.9 / 3.6) \\
n=224\end{array}$ & $\begin{array}{l}38 / 134 / 13 / 8 \\
(19.7 / 69.4 / 6.7 / 4.1) \\
n=193\end{array}$ & $\begin{array}{l}6 / 19 / 6 / 0 \\
(19.4 / 61.3 / 19.4 / 0) \\
n=31\end{array}$ & 0.087 \\
\hline Pathological stage, I/II/III/IV (\%) & $\begin{array}{l}33 / 79 / 106 / 11 \\
(14.4 / 34.5 / 46.3 / 4.8) \\
n=229\end{array}$ & $\begin{array}{l}28 / 65 / 95 / 10 \\
(14.4 / 32.8 / 48 / 5.1) \\
n=198\end{array}$ & $\begin{array}{l}5 / \mid 4 / I I / I \\
(\mid 6.1 / 45.2 / 35.5 / 3.2) \\
n=3 \mid\end{array}$ & 0.506 \\
\hline Immunohistochemistry & & & & \\
\hline Ki-67, positivity (\%) & $231 / 231(100)$ & $200 / 200(100)$ & $3|/ 3|(100)$ & 1.000 \\
\hline p53, positivity (\%) & $|2| / 23 \mid(52.4)$ & $105 / 200(52.5)$ & $|6 / 3|(5 \mid .6)$ & 0.927 \\
\hline Nm23, positivity (\%) & $|40 / 23|(60.6)$ & $120 / 200(60)$ & $20 / 31(64.5)$ & 0.632 \\
\hline PABPCI, positivity (\%) & I79/23I (77.5) & $154 / 200(77)$ & $25 / 31(80.6)$ & 0.833 \\
\hline $\begin{array}{l}\text { Follow-up month } \\
\text { Duration, median (range) }\end{array}$ & $\begin{array}{l}37.9(I-93) \\
n=152\end{array}$ & $\begin{array}{l}37.9(1-93) \\
n=|3|\end{array}$ & $\begin{array}{l}38.5(I-9 \mid) \\
n=2 I\end{array}$ & 0.932 \\
\hline Outcome, death (\%) & $99 / 152(65.1)$ & $86 / 131$ (65.6) & $|3 / 2|(6 \mid .9)$ & 0.738 \\
\hline Recurrence (\%) & $81 / 139(58.3)$ & $70 / 119$ (58.8) & $\mathrm{II} / 20(55)$ & 0.748 \\
\hline
\end{tabular}

Notes: Continuous variables were analyzed using the Student's $t$ test, and categorical variables were analyzed using the $\chi^{2}$ test. The data of the variables was based on the 231 patients, except "follow-up duration", "outcome" and "recurrence". Significance analysis was two-tailed, and $p<0.05$ was considered statistically significant. * at $p<0.05$ level, ** at $p<0.01$ level.

Abbreviations: ESCC, esophageal squamous cell carcinoma; U, upper esophagus; M, middle esophagus; L, lower esophagus; LN, lymph node; DTCD, degree of tumor cell differentiation; ME, medullary type; UT, ulcerative type; MU, mushroom type; CT, constrictive type.

in the upper esophagus among men with ESCC than in women. However, there was no significant difference in tumor size, tumor differentiation, LNM or pathological stage between men and women ESCC patients.

\section{Dysregulated Expression of PAPBCI mRNA and Protein in ESCC}

The mRNA expression of PABPC1 among total ESCC tissues was lower compared to paired normal tissues 
(median 0.467 vs 0.448), but it was non-significant (Figure 1A). However, the mRNA expression of PABPC1 was significantly increased among ESCC tissues with high PABPC1 expression but it was decreased in ESCC tissues with low PABPC1 expression, compared to paired normal tissues (median 0.927 vs 0.448 and median 0.332 vs 0.448 , respectively) (Figure $1 \mathrm{~B}$ ).

In contrast to findings at the mRNA level, the protein expression of PABPC1 was found to be significantly up-regulated across most ESCC tissues by the IHC assay, compared to paired normal tissues $(\mathrm{P}<0.001)$. Among the 231 ESCC cases, 179 (77.5\%) ESCC tissues exhibited positive staining within the cytoplasm, while only $12(5.2 \%)$ paired normal tissues exhibited positive staining $(\mathrm{P}<0.001)$. The average percentage of PABPC1 expression in the ESCC tissues from patients with pathological stage (pStage)-I, -II, -III, and -IV ESCC was $31.5 \%( \pm 28.9 \%), 32.0 \%$ ( $\pm 30.6 \%), 42.8 \%$ ( \pm $31.2 \%$ ), and $38.2 \%$ ( $\pm 32.6 \%)$, respectively. Overall, 95 (41.1\%) ESCC tissues exhibited high expression of PABPC1. The percentage of ESCC tissues with high expression of PABPC1 from patients with pStage-I, -II,
-III, and -IV ESCC was 35.3\%, 31.6\%, 50.5\%, and $45.5 \%$, respectively.

\section{Association of PABPCI Expression with Clinicopathological Characteristics in Patients with ESCC}

In ESCC, the IHC staining of PABPC1 was limited only to the cytoplasm of the ESCC cells (Figure 2A-F). PABPC1 protein expression was significantly correlated with LNM $(\mathrm{P}=0.011)$, pathological stage $(\mathrm{P}=0.021)$, tumor recurrence $(\mathrm{P}<0.001)$, and clinical outcome of the patients with ESCC $(\mathrm{P}<0.001)$. However, PABPC1 protein expression was not found to be correlated with age, gender, smoking or drinking habit, tumor site, tumor size, tumor differentiation, and pathological type (Table 2). High percentage of PABPC1-positive tumor cells in ECSS was closely associated with poor survival, while the percentage of positive tumor cells in ECSS of any of the three molecular markers Ki-67, p53 and nm23 revealed no correlation with the outcome of patients with ESCC. Moreover, the expression of $\mathrm{Ki}$ -
A

${ }^{4.0} 7$

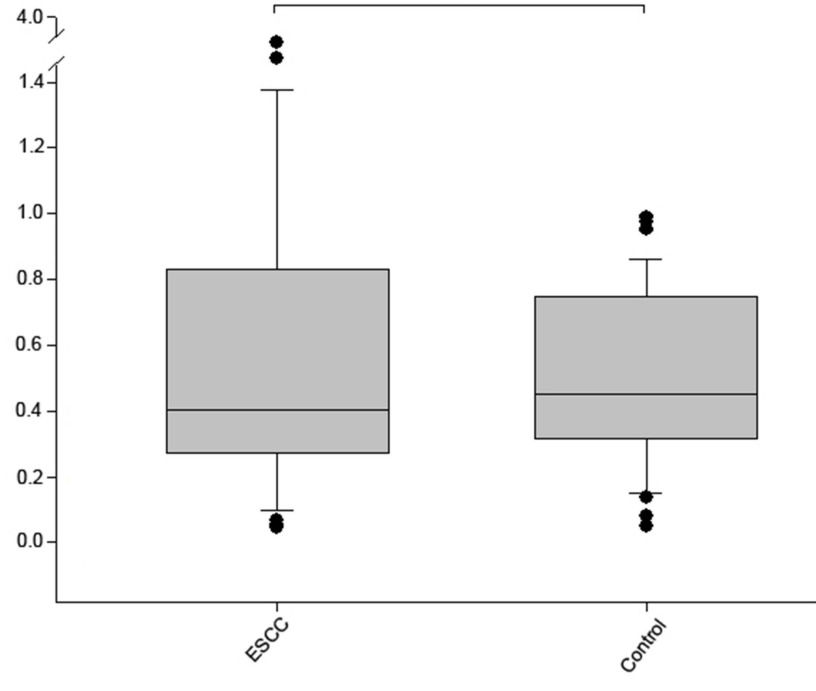

B

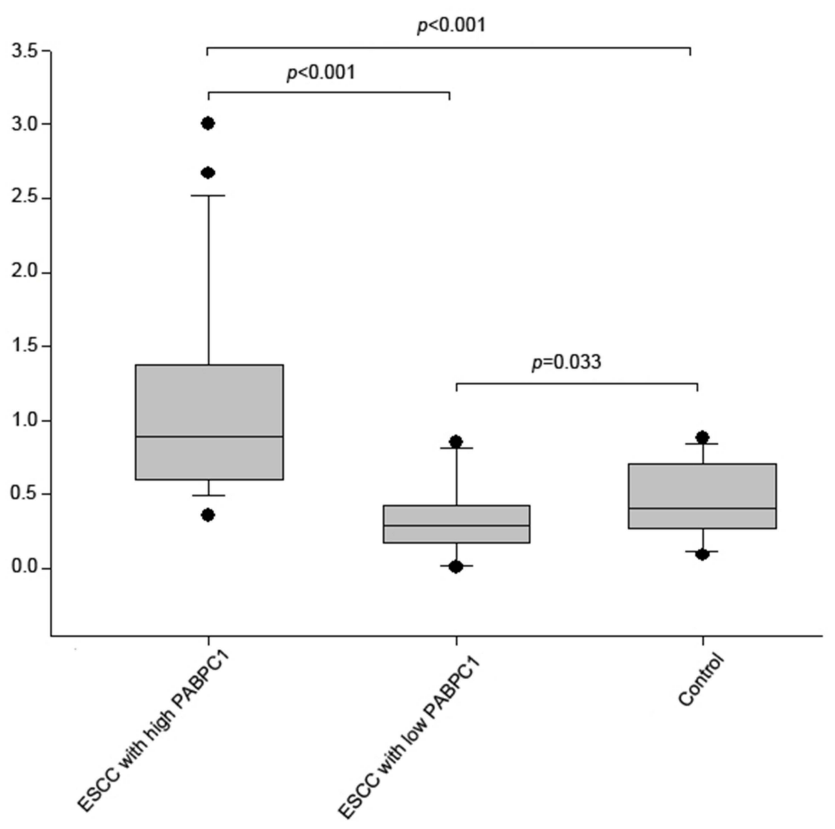

Figure I The mRNA expression level of PABPCI in ESCC.

Notes: The mRNA expression levels of PABPCI in total ESCC and their paired adjacent normal tissues (control) (A); the mRNA expression level of PABPCI was increased in ESCC with high PABPCI expression, but decreased in ESCC with low PABPCI expression, compared to that of the control (B). Student's $t$ test was used for significance analysis (two-tailed), and $p<0.05$ was considered statistically significant. Abbreviation: ESCC, esophageal squamous cell carcinoma. 


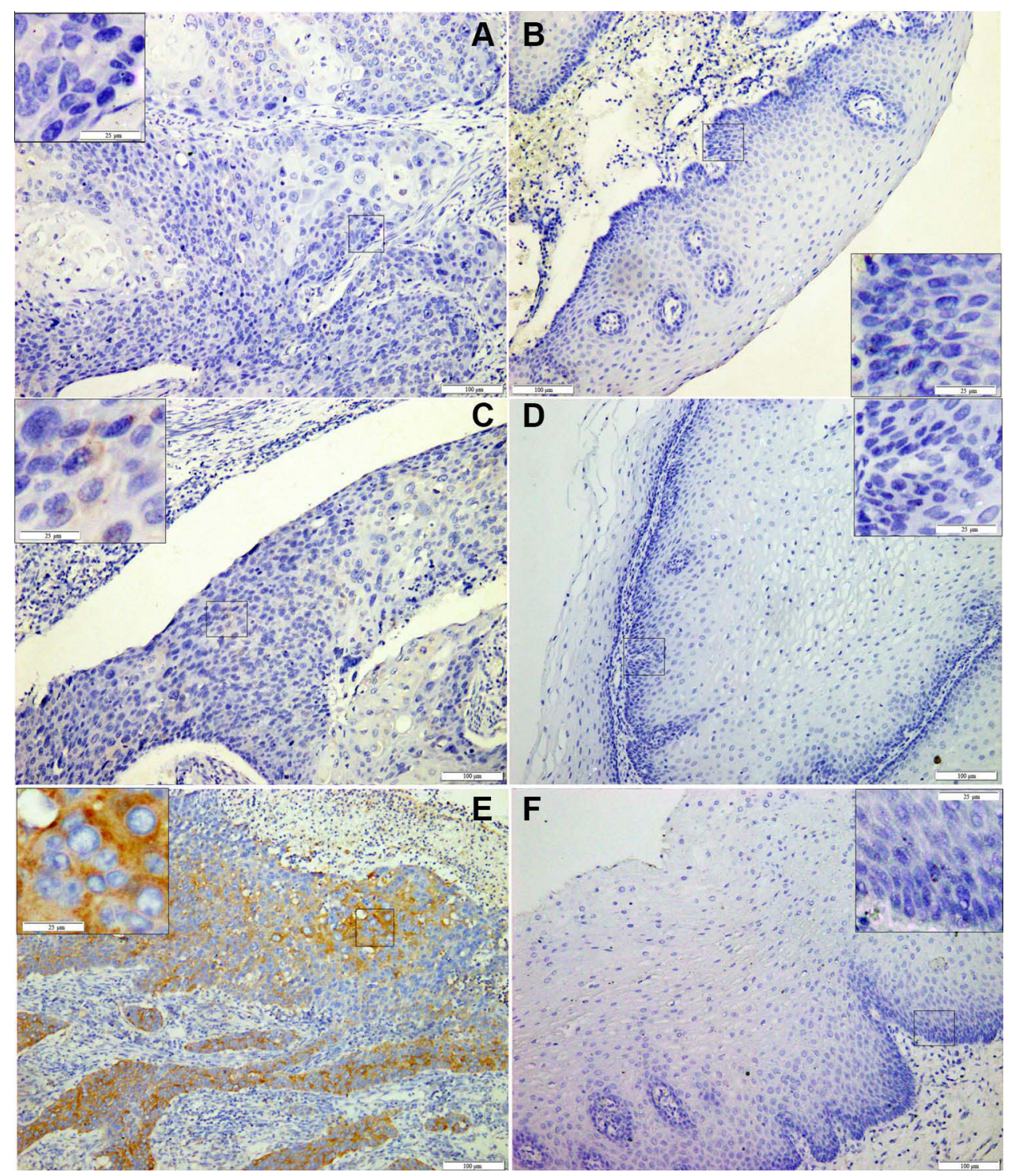

Figure 2 Immunohistochemical staining of PABPCI in ESCC.

Notes: Representative images of PABPCI staining with low expression $(\mathbf{A}, \mathbf{C})$ and high expression $(\mathbf{E})$ in ESCC tissues and their paired adjacent normal epithelium tissues (B,D, F, respectively). Abbreviation: ESCC, esophageal squamous cell carcinoma.

67, p53, or nm23 was not correlated with that of PABPC1 protein.

\section{High PABPCI Expression Was Associated with Poor Overall Survival Time}

Among the 231 patients, the complete follow-up data were available for $152(65.8 \%)$ ESCC patients with a median follow-up period of 41.7 months (range 0-96 months). Of these 152 patients, $54(35.5 \%)$ patients were alive, including 51 (94.4\%) without ESCC keep and $3(5.6 \%)$ with. Kaplan-Meier survival analysis revealed that PABPC1 expression was significantly correlated with the outcome of ESCC patients after surgery. Patients with low expression of PABPC1 had a significantly better OS than those with high expression $(\mathrm{P}<0.001)$ (Figure $3 \mathrm{~A})$. The median survival time in low and high expression groups was 55.5 $( \pm 34.5)$ and $25.9( \pm 26.2)$ months, respectively. The 5-year survival of patients with pStage-I, -II, -III, and -IV ESCC with low PABPC1 expression was 100\%, 71.9\%, 24.1\%, and $0 \%$, respectively. On the other hand, the 5-year survival of patients with high PABPC1 expression was $12.5 \%$, $21.1 \%, 5.0 \%$, and $0 \%$, respectively. Relationships between the expression of $\mathrm{Ki}-67, \mathrm{p} 53$, and $\mathrm{nm} 23$ and the prognosis of patients with ESCC were also analyzed. However, no 


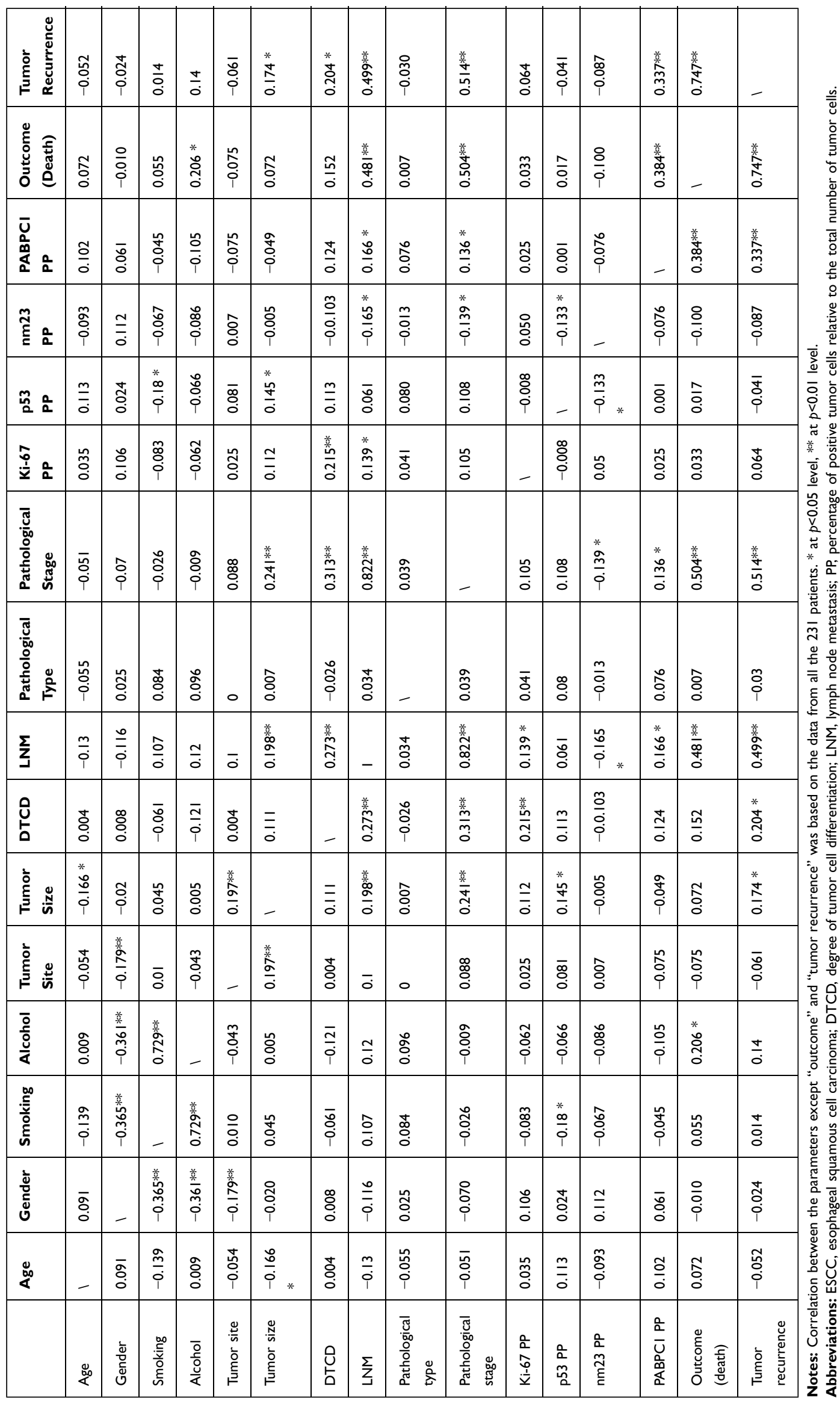



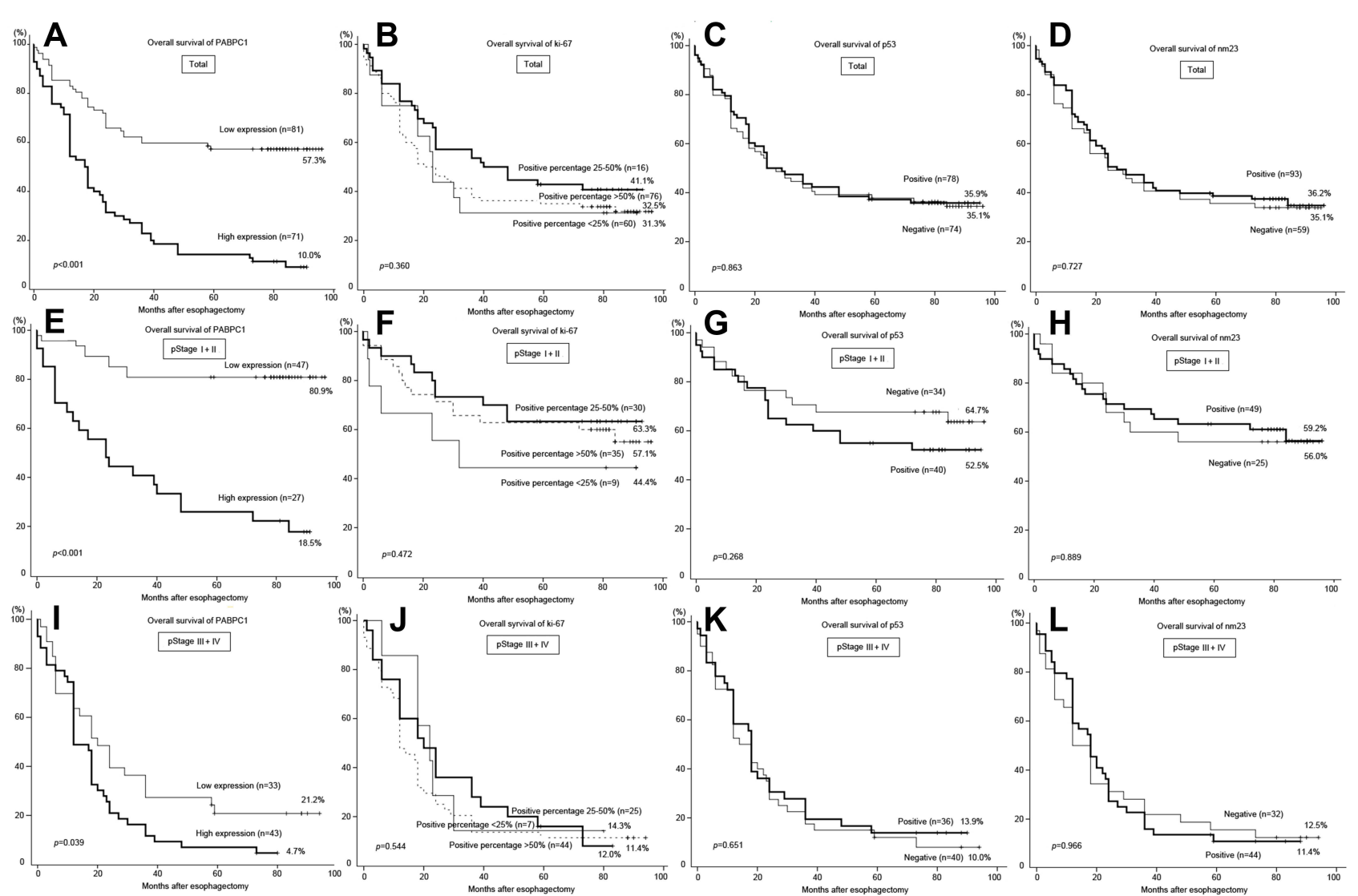

Figure $3 \mathrm{Ki}-67, \mathrm{p} 53, \mathrm{~nm} 23$, and PABPCI in the outcome prediction of the patient with ESCC.

Notes: Kaplan-Meier analyses revealed that high expression of PABPCI was correlated with poor OS in total ESCC specimens (A); expression of Ki-67, p53, and nm23 was not correlated with poor OS in total ESCC specimens (B-D); high expression of PABPCI was correlated with poor OS in early-staged (pStage-I,-II) ESCC specimens (E) and advanced (pStage-III, -IV) ESCC specimens (I); expression of Ki-67, p53, and nm23 was not correlated with poor OS in early-staged (pStage-I,-II) ESCC specimens (F, G, H, respectively) or advanced (pStage-III, -IV) ESCC specimens (J, K, L, respectively). Abbreviations: ESCC, esophageal squamous cell carcinoma; OS, overall survival.

significant correlation was observed between OS and expression of any of the three protein markers (Figure 3B-D).

Notably, PABPC1 exhibited an improved prognosis among early-stage ESCC compared to advanced ESCC. The survival rate of patients with low expression of PABPC1 in pStage-I and -II ESCC was $80.9 \%$, which is much higher than that of ESCC with high expression of PABPC1 (18.5\%) (Figure 3E). On the other hand, the survival rate of patients with low expression of PABPC1 in pStage-III and -IV ESCC was down to $21.2 \%$, which is still significantly higher compared to patients with high expression of PABPC1 $(\mathrm{P}=0.039)$ (Figure 3I). The expression of $\mathrm{Ki}-67, \mathrm{p} 53$, and $\mathrm{nm} 23$ did not exhibit any prognostic function, regardless of early- or advanced ESCC (Figure 3F-H and J-L).

\section{Analysis of Prognostic Factors Using Univariate and Multivariate Cox Regression Analysis}

Cox regression was performed to evaluate the association of significant risk factors with OS in ESCC patients. The results of Univariate analysis indicated that age $(\mathrm{P}=0.781)$, gender $\quad(\mathrm{P}=0.854)$, smoking $\quad(\mathrm{P}=0.788)$ or drinking $(\mathrm{P}=0.182)$ were not found to be statistically significant risk factors for OS of patients with ESCC (Table 3). The regions of the middle and lower esophagus were associated with a more favorable outcome $(\mathrm{P}=0.008$ and 0.014 , respectively). ESCC patients with poorly differentiated tumors showed 2.535-fold (95\% CI 1.247-5.156; $\mathrm{P}=0.01$ ) increased risk of mortality than highly differentiated tumors. Metastasis of lymph node was a significant 
Table 3 Analysis of Prognostic Factors Using Univariate and Multivariate Analyses Among the I52 Followed-Up ESCC Patients

\begin{tabular}{|c|c|c|c|c|}
\hline \multirow[t]{2}{*}{ Variable } & \multicolumn{2}{|l|}{ Univariate } & \multicolumn{2}{|l|}{ Multivariate } \\
\hline & HR $(95 \% \mathrm{CI})$ & $P$ & HR $(95 \% \mathrm{CI})$ & $P$ \\
\hline Age $(y),>60$ & $1.058(0.71 \mathrm{I}-1.574)$ & $0.78 I$ & & \\
\hline Gender, man & $0.947(0.528-1.697)$ & 0.854 & & \\
\hline Smoking, $>5 /$ day & $1.058(0.703-1.592)$ & 0.788 & & \\
\hline Alcohol, $>25$ g/day & $1.320(0.878-1.985)$ & 0.182 & & \\
\hline Tumor site, $U$ & I & & I & \\
\hline$M$ & $0.351(0.163-0.758)$ & $0.008^{* *}$ & $0.301(0.13-0.696)$ & $0.005^{* *}$ \\
\hline L & $0.380(0.176-0.82)$ & $0.014 *$ & $0.264(0.114-0.611)$ & $0.002^{* *}$ \\
\hline Tumor size, $\leq 3 \mathrm{~cm}$ & I & & & \\
\hline $3-5 \mathrm{~cm}$ & $1.260(0.823-1.929)$ & 0.287 & & \\
\hline$>5 \mathrm{~cm}$ & $1.333(0.614-2.893)$ & 0.467 & & \\
\hline DTCD, Well & 1 & & I & \\
\hline Moderate & $1.338(0.88-2.033)$ & 0.173 & $0.964(0.47 I-1.974)$ & 0.483 \\
\hline Low & $2.535(1.247-5.156)$ & $0.010 *$ & $1.002(0.499-2.011)$ & 0.737 \\
\hline No. of LNs metastasized, 0 & I & & I & \\
\hline $1-2$ & $3.274(2.052-5.223)$ & $<0.001 * *$ & $1.664(0.784-3.532)$ & 0.185 \\
\hline$\geq 3$ & 4.637 (2.688-7.999) & $<0.001 * *$ & $2.593(1.038-6.476)$ & $0.04 I^{*}$ \\
\hline Pathological type, ME & I & & & \\
\hline UT & $1.566(0.898-2.732)$ & 0.114 & & \\
\hline MU & $1.282(0.466-3.531)$ & 0.63 & & \\
\hline CT & $0.608(0.139-2.661)$ & 0.509 & & \\
\hline Pathological stage, I & I & & 1 & \\
\hline II & $1.582(0.68 \mathrm{I}-3.675)$ & 0.286 & $1.272(0.53-3.049)$ & 0.590 \\
\hline III & 5.591 (2.389-13.085) & $<0.001 * *$ & $2.235(0.79-6.324)$ & 0.130 \\
\hline IV & $7.934(2.216-28.403)$ & $0.001 * *$ & $3.645(0.847-15.678)$ & 0.082 \\
\hline $\mathrm{Ki}-67,0-25 \%$ & I & & & \\
\hline $25-50 \%$ & $0.952(0.537-1.685)$ & 0.865 & & \\
\hline$>50 \%$ & $1.040(0.579-1.867)$ & 0.895 & & \\
\hline P53, 0-25\% & I & & & \\
\hline $25-50$ & $0.642(0.294-1.398)$ & 0.264 & & \\
\hline $50-75$ & $1.294(0.665-2.518)$ & 0.448 & & \\
\hline$>75 \%$ & $0.848(0.484-1.487)$ & 0.565 & & \\
\hline $\mathrm{nm} 23,0-25 \%$ & 1 & & & \\
\hline $25-50 \%$ & $0.945(0.557-1.602)$ & 0.833 & & \\
\hline $50-75 \%$ & $0.748(0.382-1.467)$ & 0.398 & & \\
\hline$>75 \%$ & $0.625(0.34|-| .144)$ & 0.127 & & \\
\hline PABPCI, low expression & 1 & & 1 & \\
\hline PABPCI, high expression & $3.178(2.092-4.829)$ & $<0.00 I^{* *}$ & $2.622(1.68-4.129)$ & $<0.00 I^{* *}$ \\
\hline
\end{tabular}

Notes: Significance analysis was two-tailed, and $p<0.05$ was considered statistically significant. $*$ at $p<0.05$ level, $* *$ at $p<0.01$ level.

Abbreviations: ESCC, esophageal squamous cell carcinoma; U, upper esophagus; M, middle esophagus; L, lower esophagus; DTCD, degree of tumor cell differentiation; LN, lymph node; ME, medullary type; UT, ulcerative type; MU, mushroom type; CT, constrictive type. 
independent risk factor for poor OS $(\mathrm{P}<0.001)$, irrespective of the number of sites of metastasis. ESCC patients with pStage-III and -IV had over 5-fold increased risk of unfavorable survival than ESCC patients with pStage-I; however, no statistical significance was found between the two stages. High expression of PABPC1 was a significant predictor of poor OS. ESCC patients with high expression of PABPC1 protein had 3.178-fold (2.092-4.829) increased risk of unfavorable OS than ESCC patients with low expression $(\mathrm{P}<0.001)$. The expression of Ki-67, p53, or nm23 was found to not be an independent risk factor for poor OS compared to PABPC1 protein expression in patients with ESCC.

Significant factors were then entered into the multivariate Cox regression analysis (Table 3). In a multivariable logistic regression model, tumor site ( $\mathrm{P}=0.005$ and 0.002), $\geq 3$ LNs metastasis (0.041), and high expression of $\mathrm{PABPC} 1$ protein $(\mathrm{P}<0.001)$ were found to be independent prognostic factors for poor OS.

\section{Discussion}

PABPC1 is the major isoform of PABP that interacts with poly(A) tail, and the complex primarily mediates the pathways responsible for RNA polyadenylation, translation initiation, and mRNA stabilization. ${ }^{17}$ In this study, we reported that dysregulated expression of PABPC1 was associated with ESCC. ESCC patients with high PABPC1 protein expression exhibited a poor prognosis. Therefore, the up-regulation of PABPC1 may contribute to tumor growth in patients with ESCC by regulating mRNA turnover. However, Takashima et al documented that the mRNA expression level of $P A B P C 1$ was significantly downregulated in advanced ESCCs and was correlated to poor survival. ${ }^{13}$ We also discovered that the mRNA expression of PABPC1 was downregulated in ESCC tissues with low expression of PABPC1, but it was significantly upregulated in ESCC tissues with high expression of PABPC1. Actually, two specimens (from patient 6 and 17) exhibited an extremely high ratio of $P A B P C 1 / G A P D H$ in the study of Takashima et al, which was in accordance with our results, which show upregulation of $P A B P C 1$ mRNA in ESCC tissues with high expression of PABPC1 and down-regulation of PABPC1 mRNA in ESCC tissues with low expression of PABPC1. Additionally, high expression of PABPC1 was correlated with unfavorable prognosis, which indicated that $P A B P C 1$ functions as a tumor promoting gene in at least part of the ESCCs.

Expression of the $\mathrm{Ki}-67$ protein, a well-established marker of proliferation during G1 and S phases, was used to detect the malignant growth of epithelial tumor cells. ${ }^{18-20}$ Ki-67 index of the normal esophageal epithelium was lower than that of ESCC, and that of dysplasia ${ }^{21}$ In the present study, positive Ki-67 immunostaining was presented in all the 231 ESCC cases; however, no positive immunostaining was detected in paired normal tissues, indicating that increased proliferative activity in ESCC cells. However, no statistical significance for OS was observed between ESCC patients with high Ki-67 index and low Ki-67 index in this study. This finding was in accordance with the results from three eligible studies assessing $\mathrm{Ki}-67$ in ESCC that revealed an HR of 1.11 (95\% CI, 0.70-1.78) with no significant heterogeneity. ${ }^{22}$ Inactivation of the p53 tumor suppressor, a crucial tumor suppressor and a master regulator of many signaling pathways, is the most prevalent event in human cancers. Several studies have indicated that p53 protein accumulation is frequently associated with ESCC tumors and precancerous lesions, ${ }^{23}$ in contrast to the other tumor suppressor p16, which is a critical regulator of G1 phase cell cycle arrest and senescence and the most frequent target of epigenetic inactivation in human cancers. ${ }^{24} \mathrm{We}$ detected p53 accumulation in over $50 \%$ of ESCC tissue specimens, similar to the result of Ye et al in ESCC, ${ }^{25}$ revealing the extensive presence of p53 mutations in ESCC. Nevertheless, p53 expression was not correlated with Ki-67 index or the outcome of patients with ESCC but was correlated with a smoking habit, consistent with the findings of Taghavi et al. ${ }^{15}$ Thus, we anticipated that p53 combined with other molecular markers rather than p53 alone represented a better strategy to assess the prognosis of patients with ESCC. Unlike Ki-67 and p53, the NM23 gene, a metastasis suppressor gene, is defined by its capacity to regulate the metastatic dissemination in vivo without influencing the tumor growth. ${ }^{26}$ In this study, positive expression of $\mathrm{nm} 23$ protein was observed in 140 ESCC specimens (60.6\%), and its high expression was significantly negatively correlated with LNM, pathological stage, and p53 expression, but not with the outcome of patients with ESCC. The HR of $\mathrm{nm} 23$ for OS was not found to be significant between high and low expression groups, confirming that $\mathrm{nm} 23$ played an important role in the occurrence of distant metastasis in esophageal 
cancer. ${ }^{27}$ However, this is not an independent factor for prognosis of patients with ESCC.

In this study, we identified a cytoplasmic protein PABPC1 with specific expression in ESCC as detected by IHC. The expression of PABPC1 was significantly correlated with LNM and pathological stage, but not with the tumor size or differentiation. This was similar to the performance of nm23, as well as several other suppressors of metastasis, ${ }^{28}$ revealing that PABPC1 is a putative predictor of metastasis. However, nm23 was not found to be an effective predictor of prognosis in this study, and Ki-67 and p53 was not. We estimated that although Ki-67, p53, and nm23 were important regulators of the tumorigenesis and progression in many tumors they did not represent the key regulatory pathways in ESCC, but PABPC1 may represent. Furthermore, the HR of PABPC1 (high/low) for OS in multivariate analysis was up to 2.622 (1.684.129) (95\% CI, $\mathrm{P}<0.001)$, which was considered to be better than those of the 13 unique ESCC biomarkers reviewed from 109 eligible studies. ${ }^{22}$

Recently, Zhang et al reported that COL11A1 was highly expressed in ESCC tumors at advanced stages, ${ }^{29}$ which was an independent prognostic factor for OS as well as for tumor stage. The high immunoreactive intensity of COL11A1 was observed in stage-II and -III tumors, but not in stage-I, implying that COL11A1 is not eligible for prognosis of early-stage ESCC. Herein, although PABPC1 expression was correlated with a pathological stage, the as high immunoreactive intensity of PABPC1 was detected in ESCC patients with stage-I tumors, we did not observe a significant difference between pStage-I and -II ESCC $(\mathrm{P}=0.464)$. Therefore, PABPC1 appeared to be an excellent prognostic biomarker for early-stage ESCC, compared to advanced ESCC. The 5-year survival of the pStage-I and -II ESCC patients with high expression of PABPC1 was found to only be $18.5 \%$, which was substantially lower than that of the same stage ESCC patients with low expression of PABPC1 (80.9\%). Our findings that $63.5 \%$ of early-stage ESCC patients exhibit low expression of PABPC1 indicate that a test of PABPC1 levels can be included as a triage strategy, and can help over $60 \%$ of early-stage ESCC patients avoid excessive treatment. Comparatively, the 5-year survival of patients with advanced ESCC was extremely bad, regardless of whether PABPC1 expression in ESCC was high or low. We estimate that PABPC1 is a factor that aggravates malignance of ESCC, but may not be one of the key factors that regulate ESCC progression.

In conclusion, the results of the present study highlight the potential of PABPC1 as a promising prognostic marker and therapeutic target for ESCC, especially for early-stage ESCC. Expression of Ki-67, p53, and nm23 was correlated with the occurrence, development and metastasis of tumor cells, but is not an eligible prognostic factor for survival of ESCC patients. Additional molecular biomarkers are needed to be developed for better prognosis of advanced ESCC.

\section{Abbreviations}

AJCC, American Joint Committee on Cancer; CI, confidence interval; ESCC, esophageal squamous cell carcinoma; HR, hazard ratio; IHC, immunohistochemistry; LNM, lymph nodes metastasis; NMD, nonsense-mediated decay; OS, overall survival; PABPC1, cytoplasmic poly (A) binding protein 1; pStage, pathological stage.

\section{Acknowledgments}

This research was supported by PhD Research Fund of the Affiliated Hospital of the Southwest Medical University (grant no. 16238), Luzhou city: Southwest Medical University Joint Project (grant no. 2018LZXNYD-ZK22), and Southwest Medical University Project (grant no. 2019ZQN070).

\section{Author Contributions}

All authors contributed to data analysis, drafting or revising the article. All authors gave final approval of the manuscript to be published, agreed to the submitted journal, and agreed to be accountable for all aspects of the work.

\section{Disclosure}

The authors declare that they have no conflicts of interests in this work.

\section{References}

1. Wu YF, Chu SC, Chang BS, Cheng YT, Wang TF. Hematologic markers as prognostic factors in nonmetastatic esophageal cancer patients under concurrent chemoradiotherapy. Biomed Res Int. 2019;5:1-11.

2. Sasaki Y, Tamura M, Koyama R, Nakagaki T, Adachi Y, Tokino T. Genomic characterization of esophageal squamous cell carcinoma: insights from next-generation sequencing. World $J$ Gastroenterol. 2016;22(7):2284-2293. doi:10.3748/wjg.v22.i7.2284

3. Lin CH, Hung TM, Chang YC, et al. Prognostic value of lymph node-to-primary tumor standardized uptake value ratio in esophageal squamous cell carcinoma treated with definitive chemoradiotherapy. Cancers. 2020;12(3):607. doi:10.3390/cancers12030607 
4. Tong YS, Wang XW, Zhou XL, et al. Identification of the long non-coding RNA POU3F3 in plasma as a novel biomarker for diagnosis of esophageal squamous cell carcinoma. Mol Cancer. 2015;14:3. doi:10.1186/1476-4598-14-3

5. Ohashi S, Miyamoto S, Kikuchi O, Goto T, Amanuma Y, Muto M. Recent advances from basic and clinical studies of esophageal squamous cell carcinoma. Gastroenterology. 2015;149(7):1700-1715. doi:10.1053/j.gastro.2015.08.054

6. Vumbaca F, Phoenix KN, Rodriguez-Pinto D, Han DK, Claffey KP. Double-stranded RNA-binding protein regulates vascular endothelial growth factor mRNA stability, translation, and breast cancer angiogenesis. Mol Cell Biol. 2008;28(2):772-783. doi:10.1128/ MCB.02078-06

7. Kini HK, Silverman IM, Ji X, Gregory BD, Liebhaber SA. Cytoplasmic poly(A) binding protein-1 binds to genomically encoded sequences within mammalian mRNAs. RNA. 2016;22(1):61. doi:10.1261/rna.053447.115

8. Chorghade S, Seimetz J, Emmons R, et al. Poly(A) tail length regulates $\mathrm{PABPC} 1$ expression to tune translation in the heart. eLife. 2017;6:e24139. doi:10.7554/eLife.24139

9. Gray NK, Hrabálková L, Scanlon JP, Smith RW. Poly(A)-binding proteins and mRNA localization: who rules the roost? Biochem Soc Trans. 2015;43(6):1277-1284. doi:10.1042/BST20150171

10. Peixeiro I, Â I, Barbosa C, Silva AL, Liebhaber SA, Romão L. Interaction of PABPC1 with the translation initiation complex is critical to the NMD resistance of AUG-proximal nonsense mutations. Nucleic Acids Res. 2012;40(3):1160-1173. doi:10.1093/ nar/gkr820

11. Zhu J, Ding H, Wang X, Lu Q. PABPC1 exerts carcinogenesis in gastric carcinoma by targeting miR-34c. Int $J$ Clin Exp Pathol. 2015;8(4):3794-3802.

12. Zhang $\mathrm{H}$, Sheng $\mathrm{C}$, Yin $\mathrm{Y}$, et al. PABPC1 interacts with $\mathrm{AGO} 2$ and is responsible for the microRNA mediated gene silencing in high grade hepatocellular carcinoma. Cancer Lett. 2015;367(1):49-57. doi:10.1016/j.canlet.2015.07.010

13. Takashima N, Ishiguro H, Kuwabara Y, et al. Expression and prognostic roles of PABPC1 in esophageal cancer: correlation with tumor progression and postoperative survival. Oncol Rep. 2006;15 (3):667-671.

14. Sasagawa H, Shiozaki A, Iitaka D, et al. Ki-67 labeling index as an independent prognostic factor in human esophageal squamous cell carcinoma. Esophagus. 2012;9:195-202. doi:10.1007/s10388-0120336-6

15. Taghavi N, Biramijamal F, Sotoudeh M, et al. Association of p53/p21 expression with cigarette smoking and prognosis in esophagealsquamous cell carcinoma patients. World J Gastroenterol. 2010;16 (39):4958. doi:10.3748/wjg.v16.i39.4958

16. Indinnimeo M, Cicchini C, Stazi A, et al. nm23-H1 protein expression in anal canal carcinoma: does it correlate with prognosis? J Surg Oncol. 2000;74(2):163-166. doi:10.1002/1096-9098(200006) 74:2<163::AID-JSO16>3.0.CO;2-D
17. Cragle CE, MacNicol MC, Byrum SD, et al. Musashi interaction with poly(A)-binding protein is required for activation of target mRNA translation. J Biol Chem. 2019;294(28):10969-10986. doi:10.1074/ jbc.RA119.007220

18. Sun XM, Kaufman PD. Ki-67: more than a proliferation marker. Chromosoma. 2018;127(2):175-186. doi:10.1007/s00412-018-06598

19. Heayn M, Skvarca LB, Zhu L, et al. Impact of Ki-67 labeling index on prognostic significance of the chemotherapy response score in women with tubo-ovarian cancer treated with neoadjuvant chemotherapy. Int $J$ Gynecol Pathol. 2021;40(3):278-285. doi:10.1097/PGP.0000000000000706

20. Giurgea LN, Ungureanu C, Mihailovici MS. The immunohistochemical expression of p53 and Ki67 in ovarian epithelial borderline tumors. Correlation with clinicopathological factors. Rom J Morphol Embryo. 2012;53(4):967.

21. Kawakubo H, Ozawa S, Ando N, et al. Alterations of p53, cyclin D1 and $\mathrm{pRB}$ expression in the carcinogenesis of esophageal squamous cell carcinoma. Oncol Rep. 2005;14(6):1453-1459.

22. Chen M, Huang J, Zhu Z, Zhang J, Li K. Systematic review and meta-analysis of tumor biomarkers in predicting prognosis in esophageal cancer. BMC Cancer. 2013;13:539. doi:10.1186/1471-2407-13539

23. Saeki H, Ohno S, Miyazaki M, et al. p53 protein accumulation in multiple oesophageal squamous cell carcinoma: relationship to risk factors. Oncology. 2002;62(2):175-179. doi:10.1159/000048264

24. Taghavi N, Biramijamal F, Sotoudeh M, et al. p16 ${ }^{\text {INK4a }}$ hypermethylation and p53, p16 and MDM2 protein expression in Esophageal Squamous Cell Carcinoma. BMC Cancer. 2010;10(1):138. doi:10.1186/1471-2407-10-138

25. Ye J, Zhang L, Li Z, et al. High expression of MDM2 and the p53 protein is predictive biomarkers for poor prognosis of oesophageal squamous cell carcinoma. Cancer Manag Res. 2021;13:2733-2744. doi:10.2147/CMAR.S280326

26. Khan I, Steeg PS, The relationship of NM23 (NME) metastasis suppressor histidine phosphorylation to its nucleoside diphosphate kinase, histidine protein kinase and motility suppression activities. Oncotarget. 2018;12:10185-10202. doi:10.18632/oncotarget.23796

27. Guo XQ, Li XY. The expression and clinical significance of metastasis suppressor gene and matrix metalloproteinase-2 in esophageal squamous cell of carcinoma. Pak J Pharm Sci. 2016;29(4 Suppl): 1339 .

28. Steeg PS, Ouatas T, Halverson D, Palmieri D, Salerno M. Metastasis suppressor genes: basic biology and potential clinical use. Clin Breast Cancer. 2003;4(1):51-62. doi:10.3816/CBC.2003.n.012

29. Zhang B, Zhang C, Yang X, et al. Cytoplasmic collagen XI $\alpha$ I as a prognostic biomarker in esophageal squamous cell carcinoma. Cancer Biol Ther. 2018;19(5):364-372. doi:10.1080/ 15384047.2018.1423915

\section{Publish your work in this journal}

Cancer Management and Research is an international, peer-reviewed open access journal focusing on cancer research and the optimal use of preventative and integrated treatment interventions to achieve improved outcomes, enhanced survival and quality of life for the cancer patient.
The manuscript management system is completely online and includes a very quick and fair peer-review system, which is all easy to use. Visit http://www.dovepress.com/testimonials.php to read real quotes from published authors. 